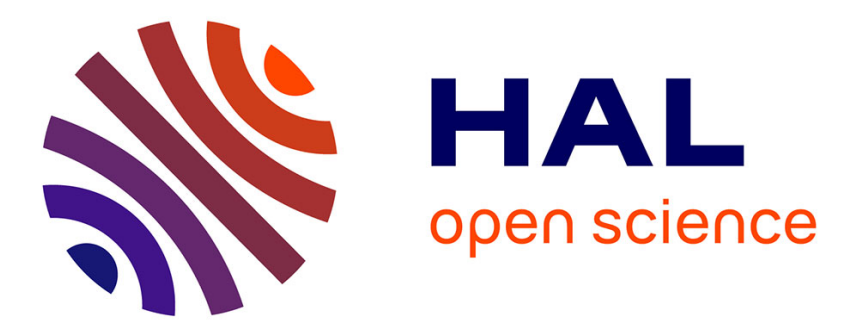

\title{
L'expérience de la Première Guerre mondiale et son influence sur la théologie dialectique du jeune Karl Barth
}

Kévin Buton-Maquet

\section{> To cite this version:}

Kévin Buton-Maquet. L'expérience de la Première Guerre mondiale et son influence sur la théologie dialectique du jeune Karl Barth. Nouvelle revue théologique, 2017, 2017/4 (139), pp.548-570. hal01778400

\section{HAL Id: hal-01778400 \\ https://univ-lyon3.hal.science/hal-01778400}

Submitted on 25 Apr 2018

HAL is a multi-disciplinary open access archive for the deposit and dissemination of scientific research documents, whether they are published or not. The documents may come from teaching and research institutions in France or abroad, or from public or private research centers.
L'archive ouverte pluridisciplinaire HAL, est destinée au dépôt et à la diffusion de documents scientifiques de niveau recherche, publiés ou non, émanant des établissements d'enseignement et de recherche français ou étrangers, des laboratoires publics ou privés. 


\title{
L'expérience de la Première Guerre mondiale et son influence sur la théologie dialectique du jeune Karl Barth
}

Kévin Buton-Maquet

\begin{abstract}
Résumé.
La guerre a bien eu une influence moins biographique que théologique sur Barth. L'expérience de la guerre rend manifeste l'incapacité des êtres humains à surmonter par leurs propres forces l'épreuve de la contradiction. Plusieurs conséquences s'ensuivent pour la dialectique barthienne. Cette dernière est une dialectique brisée, dont la contradiction logique entre la thèse et l'antithèse n'est susceptible d'aucune synthèse dans l'ordre de la pensée, Dieu étant une réalité extra-logique qui s'offre sur le mode de l'événement.
\end{abstract}

L'influence de la Première Guerre mondiale sur la pensée théologique du pasteur rouge de Safenwil fait l'objet d'évaluations divergentes. Pour certains ${ }^{1}$, cette influence serait plutôt à minimiser, dans la mesure où elle relèverait davantage d'une lecture rétrospective de l'événement par l'intéressé. La guerre servirait plutôt de révélateur et d'accélérateur d'une « crise de la prédication » par laquelle le théologien se trouverait démuni face aux «réalités sociales les plus humbles et les plus vitales ${ }^{2} »$. Il en ressort l'image d'un Barth s'opposant avant tout à l'académisme théologique de ses maîtres au nom d'une meilleure prise en compte de la réalité du monde par une théologie renouvelée. La césure importante serait donc plutôt à rechercher vers 1911, date à laquelle il rejoint la paroisse de Safenwil et découvre les réalités du milieu ouvrier.

\footnotetext{
${ }^{1}$ P. GISEL, «Première Guerre mondiale et apories de la modernité », in M. Boss, D. LAX et J. RichARD (dir.), Mutations religieuses de la modernité tardive. Actes du XIV colloque international Paul Tillich, Marseille, 2001, Münster, LIT, pp. 50-77.

${ }^{2}$ D. MÜLLER, Karl Barth, Paris, Cerf, 2005, p. 54.
} 
Pour d'autres ${ }^{3}$, et sans que l'on puisse négliger les facteurs de tension antérieurs à 1914, la Grande Guerre constitue néanmoins une véritable rupture dans le parcours intellectuel barthien. Elle est une pierre d'achoppement qui provoque un tournant qui n'aurait peut-être pas eu lieu (ou bien plus tard) face à la théologie libérale de ses maîtres. Mais même si l'on reconnaît l'importance de l'événement de la guerre sur la pensée de Karl Barth, il n'en demeure pas moins que le rôle précis de celle-ci dans sa première théologie est somme toute méconnu et ouvert à plusieurs hypothèses.

Une première hypothèse est minimaliste. Elle se contente de faire jouer à la Première Guerre mondiale un rôle strictement biographique : le conflit est l'occasion pour Barth de prendre conscience d'une faillite de la théologie de son époque (c'est-à-dire au fond, de la théologie du XIX ${ }^{\mathrm{e}}$ siècle dont Schleiermacher est le représentant le plus emblématique); il le conduit à un retour à la Bible et à une refonte de sa théologie. Mais au-delà de cet « occasionnalisme » de la guerre, celle-ci ne jouerait pas de rôle théologique majeur dans les écrits barthiens de cette époque. Il faudra attendre deux décennies, la venue d'une seconde conflagration et la menace d'une troisième, pour que la guerre devienne l'objet d'une réflexion théologique explicite dans le troisième volume de la Kirchliche Dogmatik.

Nous souhaiterions défendre, au contraire, une hypothèse maximaliste. Selon celle-ci, la guerre joue un rôle non seulement biographique mais théologique dans les premières œuvres de Barth (des prédications de guerre aux deux éditions du Römerbrief). Il est indéniable qu'elle n'y est pas présente en tant qu'objet, mais il pourrait se faire qu'elle y soit manifestée en tant qu'expérience. La guerre comme expérience révélerait ainsi la méchanceté foncière du monde et son éloignement radical vis-à-vis de Dieu. L'irruption de la guerre pourrait donc être constitutive de la «crise » (krisis) par laquelle est manifestée la « différence qualitative infinie » entre Dieu et l'être humain. Elle remplirait ainsi une fonction précise dans la logique dialectique du jeune Barth, dessinant une approche d'autant plus originale qu'elle sera résorbée plus tard au profit d'une perspective beaucoup plus classique et, il faut bien l'admettre, moins originale dans la Kirchliche Dogmatik.

Afin de tester cette hypothèse, nous évaluerons dans un premier temps l'importance psychologique et biographique de la guerre pour Barth, notamment en comparant les lettres et

\footnotetext{
${ }^{3}$ B. MCCoRmack, Karl Barth's Critically Realistic Dialectical Theology: Its Genesis and Development, 19091936, Oxford, Clarendon Press, 1995, p. 78.
} 
les prédications datant de cette époque avec les récits autobiographiques postérieurs qui reviendront sur l'événement. Nous serons alors mieux à même, dans un deuxième temps, de clarifier la façon dont on peut comprendre la guerre dans son œuvre de jeunesse, c'est-à-dire non pas comme un objet d'étude constitué par le théologien mais comme une expérience vécue. Nous pourrons enfin nous consacrer à l'analyse du rôle dialectique de la guerre et de la « ligne de mort » dans la seconde édition du Römerbrief.

\section{LE « CRÉPUSCULE DES DIEUX »}

La Première Guerre mondiale, dans la biographie de Karl Barth, est généralement comprise comme marquant « un choc et une rupture ${ }^{4}$ » qui le conduit à s'opposer à la théologie libérale. Mais que faut-il entendre par «théologie libérale »? Ce terme est souvent employé de manière lâche pour désigner en réalité des doctrines qui peuvent être très différentes entre elles. On peut définir brièvement le libéralisme théologique comme une doctrine qui valorise le pôle humain de la relation entre Dieu et la créature. Cette définition a trois corollaires. Premièrement, d'un point de vue moral, la créature dispose d'une certaine autonomie et d'une certaine initiative vis-à-vis de Dieu. Elle est homo capax. La religion joue chez elle le rôle de soutien de la morale et de l'agir humain. Deuxièmement, d'un point de vue proprement théologique, et dans une veine kantienne, la théologie libérale préfère parler de Dieu comme d'une «idée » présente à la raison humaine (quoique selon une modalité bien particulière) plutôt que d'une réalité radicalement autre. Enfin, d'un point de vue méthodologique, cette théologie privilégie l'exégèse historico-critique qui cherche à restituer le contexte de rédaction des textes plutôt que le sens qui est véhiculé (quand elle ne se limite pas, comme Barth le déplore dans la préface à la seconde édition du Römerbrief, à des considérations strictement philologiques d'ordre propédeutique $\left.{ }^{5}\right)$.

On présente donc habituellement, et avec raison, la rupture de Barth avec la théologie libérale comme la cause de l'écriture du Römerbrief dès l'été 1915. La question est plutôt celle du rôle du premier conflit mondial dans ce processus : a-t-il réellement sorti Barth de son « sommeil libéral », ou bien n'a-t-il fait qu'accélérer un désamour déjà ancien ? Il peut être utile de revenir sur les textes autobiographiques dans lesquels Barth présente lui-même la Première

\footnotetext{
${ }^{4}$ P. GiSEL, « Première Guerre mondiale et apories de la modernité », op. cit., p. 50.

${ }^{5}$ K. BARTH, L'Épître aux Romains, Pierre JUnDT (trad.), Genève, Labor et Fides, 2016, p. 14.
} 
Guerre mondiale comme l'occasion d'une rupture entre la théologie libérale et son propre parcours intellectuel :

Je me souviens en particulier d'une journée noire du début d'Août 1914. Quatre-vingttreize intellectuels allemands émurent l'opinion en en appelant à soutenir la politique militaire de Guillaume II et de ses conseillers. Parmi ces intellectuels, je découvris avec horreur la quasi-totalité de mes professeurs de théologie que je vénérais grandement. Désespéré par ce que cela signifiait pour notre temps, je compris soudainement que je ne pouvais plus désormais suivre ni leur éthique et leur dogmatique ni leur compréhension de la Bible et de l'histoire. Au moins en ce qui me concernait, la théologie du XIX ${ }^{\mathrm{e}}$ siècle n'avait plus d'avenir'.

Toutefois, ce texte date de 1957, il est donc très largement postérieur aux faits et est ainsi susceptible de recompositions inconscientes. Dans un texte encore postérieur, écrit un an avant la mort de son auteur, Barth propose un compte-rendu plus mesuré de l'influence profonde de Schleiermacher sur son propre développement intellectuel. Mais l'advenue de la guerre est décrite en des termes malgré tout très proches :

Et ce fut la Première Guerre mondiale; l'horrible manifeste des quatre-vingt-treize intellectuels allemands qui faisaient leur, au vu et au su de tout le monde, la politique guerrière de l'empereur Guillaume II et de son chancelier Bethmann-Hollweg, fut pour moi presque pire que la violation de la neutralité belge. Avec effroi, je devais découvrir parmi les signataires à peu près tous les noms de mes maîtres allemands (Martin Rade faisait une exception honorable). Tout un univers d'exégèse théologique, d'éthique, de dogmatique et d'homilétique, que j'avais tenu jusqu'alors pour authentique, fut ébranlé dans ses fondements par la lecture de ce manifeste et de ce qu'on pouvait lire à cette époque sous la plume de certains théologiens allemands ${ }^{7}$.

Malgré les nuances apportées par ce texte, tous deux partagent la même structure : (1) le déclenchement des hostilités donne libre cours au militarisme impérial ; (2) or cette politique, loin d'être condamnée par les intellectuels, se voit soutenue par la signature de l'Appel des intellectuels allemands au monde civilisé (publié le 4 octobre 1914, mais que Barth situe de manière erronée au début de la guerre); (3) ce manifeste porte (entre autres) les noms de presque

\footnotetext{
${ }^{6}$ K. BARTH, Evangelische Theologie im 19. Jahrhundert, Zollikon-Zürich, Evangelischer Verlag, 1957, p. 6, notre traduction.

${ }^{7}$ K. BARTH, « Postface sur Friedrich D. E. Schleiermacher », in La théologie protestante au dix-neuvième siècle : préhistoire et histoire, traduit par Lore JEANNERET, Genève, Labor et Fides, pp. 445-466, p. 448.
} 
tous les maîtres de Barth. Par conséquent, (4) ce dernier entérine la faillite de leur théologie, c'est-à-dire de la théologie du XIX ${ }^{\mathrm{e}}$ siècle ou de Schleiermacher.

Les récits que Barth livre rétrospectivement de l'effet de la guerre sur sa pensée sont d'une grande constance. Mais ne s'agit-il que d'une « mise en scène ${ }^{8}$ » projetée a posteriori sur les événements ? Pour répondre à cette question, l'étude de la correspondance de l'époque s'impose. Or, celle-ci nous semble confirmer dans ses grandes lignes le récit barthien : celui-ci vit bien le début de la guerre comme une crise théologique radicale. Mais certaines différences importantes n'en apparaissent pas moins.

La première différence est la suivante : dans ses écrits autobiographiques, Barth laisse penser que la lecture de l'Appel des intellectuels allemands au monde civilisé marque le point précis où les écailles lui tombent des yeux. Pourtant, c'est en réalité la lecture de la revue Christliche Welt, à laquelle il avait collaboré, qui met le feu aux poudres, avant même la lecture de l'Appel. Dès le 29 août 1914 en effet, Barth écrit une lettre à son ami Thurneysen pour se plaindre des trois dernières parutions de la revue qui viennent de lui parvenir avec du retard sans doute causé par un mois d'hostilités (une lettre antérieure au directeur de la revue Martin Rade nous apprend qu'il les attendait au moins depuis le 13 août). Après avoir découvert le contenu des trois numéros manquants, il écrit à M. Rade le 31 août :

J'ai longtemps réfléchi, je dois néanmoins maintenant vous dire à quel point les trois derniers numéros de la Christliche Welt [...] sont pour moi une déception, en termes religieux un «scandale» [« Ärgernis »]. Il m’est difficile de vous écrire cela, et cela doit vous être encore plus difficile de m'écouter. [...] La chose qui m'attriste le plus, dans cette triste période, c'est de voir comment en ce moment l'amour de la patrie, la passion pour la guerre et la foi chrétienne sont confondus de manière fatale partout en Allemagne, et comment la Christliche Welt agit au fond de la même manière que l'Allemagne toute entière. [...] nous attendions autre chose de la part de la Christliche Welt ${ }^{9}$.

Dans l'un des numéros de la revue, Rade avait réédité l'opuscule de Luther « Les soldats peuvent-ils être en état de grâce ? » précédé d'une introduction de sa main. Cette introduction évoquait la piété avec laquelle le peuple allemand se préparait à la guerre, ainsi que l'ordre dans

\footnotetext{
${ }^{8}$ P. GISEL, «Première Guerre mondiale et apories de la modernité », op. cit., p. 51.

9 Lettre du 31 août 1914, K. BARTH et M. RADE, Ein Briefwechsel, Gütersloh, Gütersloher Verlagshaus, 1981, p. 95-96, cité par C. CHALAMET, Théologies dialectiques. Aux origines d'une révolution intellectuelle, Genève, Labor et Fides, 2015, p. 103-104.
} 
lequel s'effectuait la mobilisation qui suscitait la joie céleste des anges eux-mêmes ${ }^{10}$. Il faut certes reconnaître qu'il n'y a là rien que de très classique eu égard à ce qui s'écrivait à l'époque dans le reste de l'Allemagne. Mais c'est surtout la réponse de Rade à Barth qui nous intéresse ici, car elle introduit explicitement la notion de la guerre comme expérience (Erlebnis).

Dans une lettre du 5 octobre 1914, Rade écrit à Barth que des désaccords entre eux deux ne sauraient manquer d'advenir du fait de leur différence de nationalité. Barth est Suisse, or la Suisse est un pays qui a conservé sa neutralité. La lutte n'est pas pour elle une affaire où est engagée la survie de son peuple. Rade reconnaît que cette posture permet à Barth d'atteindre un certain degré d'objectivité dans l'évaluation des responsabilités et des torts, mais elle le rend également imperméable à une autre perspective sur la guerre.

Vous pouvez raisonner paisiblement sur les points où nous sommes en faute. Vous pouvez comparer vos lectures de journaux allemands et français avec une relative certitude et parvenir à une opinion objective sur tous ces événements. Mais il y a une chose qui vous échappe : l'expérience (das Erlebnis) [...] Vous souhaitez que nous laissions Dieu en dehors de cette expérience de la guerre. Or cela est impossible. Car il n'y a qu'une seule cause et qu'un seul auteur d'une chose si puissante : Dieu ${ }^{11}$.

Il est remarquable que Rade, tout en reconnaissant que l'objectivité est du côté de son interlocuteur, n'hésite pas à considérer cette dernière comme tout à fait secondaire et même comme un obstacle en ce qu'elle empêche d'accéder à une expérience d'un genre particulier : une expérience de la guerre (et même de cette guerre) comme expression de la Providence divine. Ici encore cette mise en suspens des canons de l'objectivité scientifique n'est pas propre à Rade, mais on la trouve de manière généralisée chez les philosophes et théologiens allemands et français au début de la guerre. Tandis que la guerre était jusqu'à présent éventuellement abordée par ceux-ci comme un objet de pensée, elle devient une expérience que les intellectuels vivent en communion avec la totalité du pays. Tandis que la guerre comme objet devrait précisément se soumettre aux règles de l'objectivité, la guerre comme expérience constitue un donné originaire que la pensée conceptuelle doit s'efforcer d'élucider. De nombreux

\footnotetext{
${ }^{10}$ Die Christliche Welt $\mathrm{n}^{\circ} 33$ (15 août 1914), col. 781, cité par C. CHALAMET, Théologies dialectiques, op. cit., p. 104.

${ }^{11}$ K. BARTH et M. RADE, Bw Barth-Rade, op. cit., p. 109-110, notre traduction.
} 
philosophes et théologiens s'efforceront alors de mettre leurs théories au service de l'effort de guerre $^{12}$.

Barth perçoit la gravité d'une confusion entre expérience de la guerre et expérience de Dieu. Il ne répond pas immédiatement à Rade, mais écrit en revanche le 4 novembre 1914 à son ancien maître, Wilhelm Herrmann, pour connaître sa réaction par rapport à cette expérience de Dieu dans la guerre.

Nous avons appris de vous, monsieur le Professeur, et par votre intermédiaire des grands maîtres Luther, Kant et Schleiermacher, à voir dans «l'expérience » (Erlebnis) le principe constituant de la connaissance et de la volonté. À votre école nous avons compris ce que signifie «faire l'expérience»(erleben) de Dieu en Jésus. Or voilà que des chrétiens allemands (et récemment Rade dans sa réponse à ma lettre) nous opposent une toute nouvelle expérience soi-disant religieuse de la guerre qui devrait répondre à toutes nos réserves. Le fait que les chrétiens allemands fassent «l'expérience » de leur guerre comme d'une guerre sainte est censé nous réduire au silence et même à la révérence par rapport à cet événement. Quelle est votre position par rapport à cet argument et à la théologie de la guerre qui se cache derrière lui ? Cette « expérience » de la guerre dont parle Rade est-elle une expérience de Wotan ou une expérience de Dieu au sens chrétien ? ${ }^{13}$

Le problème que Barth commence tout juste à identifier ici n'est pas uniquement le manque d'objectivité de ses maîtres, mais leur usage de la notion d'expérience elle-même. Barth est ici sur la piste de ses découvertes théologiques futures : il est problématique de s'appuyer péremptoirement sur une expérience subjective intramondaine pour en dériver une connaissance de Dieu, car cela conduit à une confusion entre ce qui relève de Dieu et ce qui relève d'une autre réalité. Une intuition dont l'explicitation et le déploiement théologiques prendront toutefois du temps. À ce stade, l'expérience de la guerre n'est employée que comme repoussoir pour alerter de la confusion entre celle-ci et une expérience de Dieu authentique.

Ce n'est que peu avant l'envoi de cette dernière lettre à Herrmann que Barth a pris connaissance de l'Appel des intellectuels allemands au monde civilisé, que son maître lui joint dans un précédent courrier. On voit donc que la crise est déjà déclenchée chez Barth avant

\footnotetext{
${ }^{12}$ C. Prochasson et A. RASMussen, Au nom de la patrie. Les intellectuels et la Première Guerre mondiale (19101919), Paris, La Découverte, 1996.

13 Lettre à Herrmann du 4 novembre 1914, K. BARTH, Vorträge und kleinere Arbeiten (19141921), Zürich, TVZ, 2012, p. 115, cité par C. CHALAMET, Théologies dialectiques, op. cit., p. 106.
} 
même que celui-ci ne reçoive le manifeste. Mais à quel point ce document en vient-il à cristalliser les critiques de Barth contre la théologie libérale dès le début de la guerre, comme lui-même le laisse penser dans ses écrits autobiographiques ? Il semblerait que Barth, bien qu'il soit touché de plein fouet par le comportement de ses maîtres face au conflit, n'articule pas immédiatement ce comportement à une théologie particulière. Dans une lettre à W. Spoendlin du 4 janvier 1915 et revenant sur l'automne qui vient de s'écouler, il dit avoir à cette occasion vécu un « crépuscule des dieux » (Götterdämmerung) :

Les théologiens, philosophes, historiens allemands, etc., avec toutes leurs théories de la défense et de l'attaque, m'ont rendu le service de me libérer de toute politique avec eux. J'ai vécu un vrai crépuscule des dieux, en étudiant la façon dont Harnack, Herrmann, Rade, Eucken, etc., se sont positionnés vis-à-vis de cette nouvelle situation, la façon dont la religion, la science, l'art et la culture personnelle, bref tout ce que nous aimons chez les gens de l'extérieur (et avant tout chez les Allemands), se sont métamorphosés en canonnade spirituelle contre la barbarie asiatique, le mercantilisme anglais, les mensonges français, etc., et comment ils persistent encore aujourd'hui dans cette attitude. ${ }^{14}$

Dans la première phrase de l'extrait ci-dessus, Barth ne donne pas uniquement congé aux théologiens (ni a fortiori aux théologiens libéraux), mais à l'ensemble des intellectuels, considérés comme incapables de se maintenir à la hauteur de la culture qu'ils devraient défendre. Mais alors pourquoi, dans la phrase suivante pourtant, ne dénonce-t-il nommément que ses maîtres ? Sans doute parce qu'il avait placé en eux une confiance particulière. La simple expression de «crépuscule des dieux », qui évoque le titre de l'ouvrage de Nietzsche Le Crépuscule des idoles (Götzen-Dämmerung), associe l'estime vis-à-vis de ses anciens maîtres à l'adoration d'idoles. Les personnes citées n'ont pas en commun une doctrine particulière, mais une autorité spirituelle sur Barth (autorité qui s'avère usurpée, idolâtre) ${ }^{15}$.

\footnotetext{
${ }^{14}$ K. BARTH, Offene Briefe 1909-1935 (GA V.35), Zürich, TVZ, 2001, p. 22-23, notre traduction.

${ }^{15}$ On remarque, par exemple, qu'il ne nomme ni Adolf Schlatter ni Reinhold Seeberg, qui ont signé l'Appel et sont aussi les maitres de Barth (le premier fut le professeur de Barth à Tübingen, tandis que le second était professeur à Berlin, bien que Barth évita soigneusement d'aller à ses cours). Il ne les mentionne pas dans la mesure où il n'avait aucun goût pour leur conservatisme théologique, de sorte qu'il n'est pas spécialement déçu par leur conduite. Inversement, Barth jette l'anathème sur Martin Rade dans sa lettre de 1915, dont il avait été l'assistant à rédaction de la revue Die Christliche Welt, alors que celui-ci n'était pas cosignataire (Barth veillera plus tard à souligner cette «honorable exception »). Ceci semble confirmer que sa condamnation porte au-delà de la participation à l'Appel. Il ne rejette alors ni un contenu théologique précis ni même une école particulière, mais une attitude pratique inacceptable de la part d'intellectuels qu'il considérait comme des autorités spirituelles.
} 
Nous pouvons résumer nos résultats comme suit. L'analyse de certaines lettres écrites par Barth confirme le choc immense d'août 1914 dans le quotidien de Barth. Et ce choc détermina Barth à rompre avec ses maîtres, mais non pas en réponse à un manquement précis de la théologie libérale. La confusion commise par cette dernière entre expérience de Dieu et expérience de la guerre est encore perçue comme étant du même ordre que les erreurs semblables commises par la Kriegstheologie. Dans les premiers mois de la guerre, Barth réagit surtout à ce qu'il considère comme une pratique intolérable de la part de maîtres en qui il avait placé toute sa confiance. Ce n'est que plus tard qu'il articulera ce rejet à une posture théologique précise (le problème de l'ancrage de la révélation dans une expérience subjective, qui lui apparaîtra de plus en plus comme le point aveugle de la théologie libérale), ou même à un acte public précis (la signature de l'Appel).

\section{EXPÉRIENCE DE LA GUERRE, EXPÉRIENCE DE DIEU}

Dans la section précédente, nous avons résumé le rôle biographique de la Première Guerre mondiale dans la formation intellectuelle du jeune Karl Barth. Mais la guerre joue-t-elle un rôle non seulement dans sa vie, mais dans sa théologie même ? C'est pour ainsi dire un double ouvrage qui naît des ruines de la Grande Guerre : le Römerbrief de 1919 puis sa seconde édition en 1921 qui n'en laisse pas debout «pierre sur pierre ${ }^{16} »$. Plusieurs lecteurs contemporains de Barth ont pu être tentés de voir dans cette théologie de la crise le symptôme du traumatisme et du pessimisme engendré par la guerre. Mais il s'agit là d'une influence somme toute superficielle, et reposant sur une mécompréhension de la dialectique de Barth ${ }^{17}$. D'autres auteurs plus récents, afin de se prémunir contre une telle lecture, ont jugé utile d'adopter une hypothèse minimaliste vis-à-vis de l'influence de la guerre sur la théologie de Barth. Selon celle-ci, la Grande Guerre n'a qu'une importance biographique : elle lui permet de prendre conscience d'une faillite de la théologie de son époque et engendre un mouvement de réflexion qui s'émancipe bien vite de tout rapport à la guerre. La guerre ne remplirait en aucune façon une fonction théologique dans le Römerbrief.

\footnotetext{
${ }^{16}$ K. BARTH, L'Épître aux Romains, traduit par Pierre JundT, Genève, Labor et Fides, 2016, p. 10.

17 H. ZAHRNT, Aux prises avec Dieu. La théologie protestante au XXe siècle, traduit par A. LIEFOOGHE, Paris, Cerf, 1969, p. 26-27.
} 
On peut formuler deux arguments en faveur d'une telle position minimaliste. Un premier argument affirme qu'on ne trouve dans le Römerbrief de 1922 qu'un vocabulaire vestigial de la guerre. Cet argument s'appuie sur les différences entre la première et la seconde édition du Römerbrief. À la lueur de la comparaison, on s'aperçoit que les allusions à la Première Guerre mondiale s'évanouissent de l'une à l'autre. L'index de la première édition comprend une entrée «Erster Weltkrieg » qui compte dix occurrences. Ces références disparaissent intégralement dans la seconde édition. Et pour en rester à cette dernière, les expressions que l'on y trouve et qui évoquent le conflit, comme l'image fameuse des « trous d'obus » (Einschlagstrichter) pour désigner la façon dont Dieu «se rend perceptible dans la réalité visible de l'Histoire ${ }^{18}$ », semblent relever de l'ornement littéraire. En somme, il ne faudrait pas prendre trop au sérieux ce qui relèverait plutôt du style vivant adopté par l'auteur et du climat de l'après-guerre : le fond n'en serait pas touché.

Le second argument consiste à rappeler que la guerre n'est pas une notion thématisée en tant que telle dans le Römerbrief. Elle n'est pas un objet soumis aux analyses du théologien, qui se cantonne à délivrer un commentaire de l'épître de Paul aux Romains. Tout au plus peut-on admettre que la guerre joue un rôle dans l'ordre de la découverte en mettant Barth sur la piste d'une théologie dialectique renouvelée. Mais cet ordo inveniendi peut ensuite disparaître au profit de l'ordre qui est le plus conforme à la chose même (la Sache) dont s'occupe la théologie. Qui plus est, cette notion sera bien thématisée, mais beaucoup plus tard, dans la Kirchliche Dogmatik III/4. Et les commentateurs de l'éthique barthienne ne s'y sont pas trompés : aucun des principaux travaux portant sur la théorie barthienne de la guerre n'incluent une discussion du Römerbrief ${ }^{19}$.

Face au premier argument, on peut remarquer que seule la mention de la Première Guerre mondiale disparaît de l'une à l'autre édition, tandis que le champ lexical de la guerre, de la lutte et de la mort reste toujours aussi nourri. Un examen plus précis des différences entre

\footnotetext{
${ }^{18} \mathrm{~K}$. BARTH, L'Épitre aux Romains, op. cit., p. 37. Pierre Jundt se contente de traduire par «impacts », qui ne restitue à proprement parler que le premier morphème de ce syntagme. On peut également mentionner l'image de l'armistice (Waffenstillstand, p. 208, 219, 258), de la déclaration de guerre (der Krieg schon erklärt, p. 363, en référence à Lc 14, 31-32).

${ }^{19}$ J. H. YoDer, Karl Barth and the Problem of War, Nashville-New York, Abingdon Press, 1970 ; N. BIGGAR, The Hastening that Waits: Karl Barth's Ethics, Oxford, Clarendon Press, 1993, p. 92-96 ; W. WERPEHOWSKI, « Karl Barth and just War: A Conversation with Roman Catholicism», in D. Migliore (dir.), Commanding Grace: Studies in Karl Barth's Ethics, Grand Rapids, MI, Eerdmans, pp. 60-82.
} 
les deux éditions permet d'aller plus loin : non seulement ce champ lexical ne disparaît pas, mais il se trouve renforcé d'un terme supplémentaire, la « ligne de mort $^{20}$ » (Todeslinie), qui fait son apparition, massivement, dans la seconde édition alors qu'il est absent de la première. En 1922, il est difficile de penser que ce syntagme n'évoque pas à l'esprit du lecteur le no man's land entre les deux tranchées les plus avancées. Mais il s'agit bien d'un terme technique, sur lequel nous reviendrons, qui permet d'exprimer la distance infranchissable entre Dieu et l'être humain. Ce n'est donc pas une simple formule sensationnelle, mais une façon de caractériser et de décrire la relation dialectique entre Dieu et le monde. En somme, entre la première et la seconde édition, il semble que Barth prenne soin d'effacer les références explicites à la Première Guerre mondiale, peut-être afin d'éviter que l'on ne prenne son travail que pour un écrit de circonstances. Barth nourrissait en effet la crainte de n'avoir fait que dire « ce dont les oreilles démangeaient aux gens, ce qui, après la guerre, spécialement en Allemagne, était, pour ainsi dire, dans l'air ${ }^{21} »$. Mais cela n'implique pas que la guerre et la conflictualité ne jouent aucun rôle dans le Römerbrief. Notre réponse au premier argument nous met donc sur la piste d'une réponse au second : il s'agit de mieux préciser le mode de présence de la guerre dans le texte.

En réponse au second argument, il nous faut convenir que la guerre n'est pas présente dans le commentaire barthien comme un objet, c'est-à-dire comme constitué dans une analyse explicite qui viserait à en décomposer les éléments (par exemple, les différents critères qui permettent de définir une guerre comme juste ou injuste). Il s'agit encore moins de porter un discours sur l'événement historique de la Première Guerre mondiale, puisque l'auteur veille à effacer les allusions explicites à ce conflit particulier. Mais nous n'en sommes pas pour autant contraints de ne voir dans le champ lexical de la guerre qu'un résidu littéraire extérieur à la dialectique.

Notre hypothèse est que la guerre n'est pas présente dans le Römerbrief comme un objet mais à titre d'expérience. En effet, la guerre n'y est pas constituée comme objet dans une analyse explicite qui viserait à en décomposer les éléments (par exemple, les différents critères qui permettraient de définir une guerre comme juste ou injuste). Parler de guerre comme expérience désigne ici non pas ce qui est construit par l'observateur, mais ce qui s'impose à lui et face à quoi il réagit. L'expérience introduit la crise, la conversion du regard par rapport à la

\footnotetext{
${ }^{20}$ On relève onze occurrences de ce syntagme : p. 108, 117, 121,126, 134, 147, 159, 164, 168, 231 et 241 de la traduction française.

${ }^{21}$ «Préface à la cinquième édition », in K. BARTH, L'Épître aux Romains, op. cit., p. 27.
} 
réalité. Elle entraîne également la possibilité de critiquer cette réalité et de la remettre en question. Dans le cas présent, la guerre comme expérience ne désigne pas tant une pratique sociale, avec ses règles et sa limitation dans le temps, que la condition fondamentale de la réalité humaine dans son ensemble. La guerre est l'autre nom d'une expérience de la séparation radicale entre le monde et Dieu. Elle joue donc bien un rôle au sein de la dialectique barthienne, dont nous allons maintenant retracer synthétiquement les évolutions principales dans les prédications de guerre et la première édition du Römerbrief, avant d'en arriver dans un troisième moment au point sommital du Römerbrief II. Face à un certain optimisme de Römerbrief I, le retour du champ lexical de la lutte, du combat dans la seconde édition de l'ouvrage est un moyen de prendre davantage au sérieux la négativité du monde et son altérité radicale par rapport à Dieu.

\section{Les prédications de guerre.}

Nous avons vu brièvement qu'en 1914 Barth avait été choqué de trouver confondus sous la plume de ses maîtres l'expérience de Dieu et l'expérience de la guerre. L'expérience de la présence de Dieu aux côtés des combattants, le Gott mis uns de l'armée allemande, est selon lui une expérience de Wotan, autrement dit une idolâtrie. Mais dans ses prédications des premiers mois de la guerre, Barth ne sépare pas radicalement Dieu et la guerre. Il les articule plutôt : la guerre est «un temps de jugement sans pareil. Mais précisément pour cette raison également un temps de grâce tout à fait particulier ${ }^{22} »$. Ce type de déclaration est à entendre à partir d'une pensée dialectique qui est encore très proche de celle de Herrmann : il existe une contradiction au niveau de notre expérience entre le jugement de Dieu et sa grâce, entre la Loi et l'Évangile, entre le péché et la justice de Dieu. La contradiction entre ces deux pôles n'est pas susceptible d'être surmontée dans une synthèse plus haute. Le caractère dialectique de cette contradiction s'exprime en ce qu'il ne s'agit pas de choisir l'une des branches de l'alternative. Il s'agit plutôt d'habiter en chacune d'elle et de comprendre que l'une contient déjà l'autre et vice versa. Le jugement de Dieu qui condamne le pécheur contient déjà sa grâce. Réciproquement, le pécheur est sauvé par Dieu quand il est jugé. Dans le cadre de cette compréhension dialectique, Barth accepte de faire de la guerre l'expérience accablante d'un jugement qui provient de Dieu et qui est donc aussi un acte de grâce. De telles déclarations choqueront peut-être le lecteur moderne, mais dans le contexte de la surenchère patriotique du début de la guerre elles doivent être lues

\footnotetext{
${ }^{22}$ Prédication du 23 août 1914, in K. BARTH, Predigten (1914), Zürich, TVZ, 1974, p. 433, cité et traduit par C. CHALAMET, Théologies dialectiques, op. cit., p. 108.
} 
comme un refus d'une lecture qui ne verrait dans la guerre qu'un acte de grâce, voire une manifestation du divin. Barth rappelle que la guerre n'est pas une expérience positive, mais une expérience négative dans laquelle Dieu se donne à voir comme un Dieu caché. L'acte de grâce est masqué par l'expérience accablante de la mort. L'expérience de la guerre ne se donne donc en aucune façon comme une expérience qui serait susceptible d'une évaluation morale directe ; elle contient plutôt ce qui pour nous apparaît comme une contradiction qui ne devrait être appréhendée que de manière indirecte, par la voie d'une dialectique.

Il n'en demeure pas moins que Barth, au début de la guerre en tout cas, tâtonne encore quant à la compréhension dialectique de la guerre. Certaines prédications semblent même admettre une certaine continuité entre le monde livré à la guerre et l'action divine. Autrement dit, Barth peut encore passer de manière directe, univoque, c'est-à-dire non dialectique, de l'événement historique à la reconnaissance de la présence de Dieu, fut-ce sous la forme d'une présence en creux, d'une présence absente. « S'il est un moment où Barth fut tenté d'identifier l'action de Dieu aux négativités de l'histoire, c'est bien celui [de la première année de guerre $]^{23} »$. Dieu est présent dans l'événement uniquement comme le «non » opposé à toute tentative de vivre cet événement sur le mode de l'expérience religieuse. Toutefois, il y a bien la possibilité de rencontrer Dieu par l'intermédiaire d'un événement historique dans lequel il n'est certes pas directement présent et même qu'il condamne, mais qui n'en constitue pas moins une première étape vers la reconnaissance de la grâce divine.

\section{Römerbrief $I$.}

La première édition du Römerbrief tâche de mieux garantir la distinction entre Dieu et sa créature en modifiant le schéma dialectique jusqu'ici employé par Barth. Dans le but de se garder de toute univocité entre l'événement humain et l'agir divin, celui-ci veille à exacerber l'asymétrie entre les deux versants de la dialectique, qui ne sont plus équivalents. Au contraire, l'un de ces pôles est infiniment plus puissant que l'autre. Ainsi, dans les oppositions dialectiques qu'il hérite de Herrmann entre Loi et Évangile, péché et grâce, le monde et Dieu, Barth en vient à privilégier le second terme. Après 1915 se met en place ce que l'on peut appeler, avec C. Chalamet, une «dialectique supplémentaire et téléologique ${ }^{24} »$. Une dialectique est supplémentaire lorsqu'elle n'en reste pas à une tension infinie entre la thèse et

\footnotetext{
${ }^{23}$ B. MCCORMACK, Karl Barth, op. cit., p. 116.

${ }^{24}$ C. CHALAMET, Théologies dialectiques, op. cit., p. 295.
} 
l'antithèse (dialectique complémentaire), mais lorsque l'un de ces versants l'emporte sur l'autre. Cette dialectique admet dès lors une Aufhebung, dépassement de la contradiction en une synthèse plus haute ${ }^{25}$. Cette dialectique est téléologique en ce qu'elle est orientée vers la victoire de Dieu. En ce sens, le moment de la Loi n'est pas un moment autonome, le non de la créature n'est pas le mouvement premier ; au contraire, l'Évangile, le oui de Dieu sont déjà présents dans le moment critique. La résurrection est déjà présente dans le spectacle de la croix.

Il faut reconnaître dans cet infléchissement de la dialectique barthienne l'influence de Christoph Blumhardt ${ }^{26}$. En avril 1915, alors que Barth est en pleine recherche d'une orientation nouvelle à donner à sa théologie, il se rend à Bad Boll où il entend une prédication de celui-ci, condamnant le nationalisme des pays en guerre qui cherchent à rallier la religion à leurs bannières respectives, qui lui fit forte impression ${ }^{27}$. C'est de lui que Barth tiendrait la formule qui devint le mot d'ordre de sa propre théologie : «Le monde est le monde, mais Dieu est Dieu $!^{28} »$ L'un des thèmes centraux de la théologie des Blumhardt père et fils est la victoire du Christ. «Jésus est vainqueur » (Jesus ist Sieger), l'expression souligne la réalité de Dieu, au sens d'un événement qui est déjà advenu, déjà à l'œuvre en notre monde. Mais le terme martial dénote également le fait que cette réalité est radicalement opposée aux victoires humaines, à nos tentatives de confisquer la puissance de Dieu à notre profit. Il s'agit de laisser Dieu être Dieu face aux amalgames de la Kriegstheologie. Il est donc possible que la rencontre avec les Blumhardt ait non seulement (et prioritairement) mis Barth sur la voie d'une dialectique supplémentaire dans laquelle la victoire de Dieu triomphe du péché et de la mort, mais également d'une formulation de cette dialectique en des termes où l'antagonisme, pour ne pas dire la conflictualité, est particulièrement marqué.

\footnotetext{
${ }^{25}$ L'adoption d'une dialectique supplémentaire et téléologique n'est pas sans rapprocher Barth de Hegel. Toutefois, il existe des différences importantes entre leurs deux formes de dialectiques que l'on ne peut pas mentionner ici. On trouvera une mise au point dans G. DORRIEN, Kantian Reason and Hegelian Spirit: The Idealistic Logic of Modern Theology, Chichester-Malden, MA, Wiley-Blackwell, 2012, p. 454-573.

${ }^{26}$ On ne peut qu'évoquer très brièvement cette figure ici, néanmoins la recherche récente a permis de mieux en apprécier l'influence sur le développement intellectuel du pasteur rouge de Safenwil. Cf. C. Collins WINN, «Jesus Is Victor! »: The Significance of the Blumhardts for the Theology of Karl Barth, Eugene, OR, Pickwick Publications, coll. «Princeton Theological Monograph Series », n 93, 2009, p. 155-207.

${ }^{27}$ Lettre du 21 avril 1915, K. BARTH et M. RADE, Bw Barth-Rade, op. cit., p. 131.

${ }^{28}$ E. THURNEYSEN, «Théologie et socialisme dans ses lettres de jeunesse », in P. GiSEL (dir.), Karl Barth. Genèse et réception de sa théologie, Genève, Labor et Fides, pp. 121-148, p. 124.
} 
Dans le texte de 1919 pourtant, ce second aspect agonistique de la dialectique est encore largement recouvert par les accents triomphalistes du versant positif. Si la victoire du Christ est irrévocable et définitive, «si l'élection est toujours première et originelle ${ }^{29}$ », il ne convient plus de prendre la lutte trop «au tragique », pour ainsi dire. Le versant critique, négatif de la contradiction n'est qu'un contretemps avant la pleine effectuation de la synthèse. La tentative barthienne de placer la victoire de Dieu au-dessus de toute dialectique complémentaire du oui et du non le conduit à considérer cette victoire comme déjà effective, ou du moins en voie d'effectuation dans l'histoire. «Dans le courant de la soi-disant histoire est rendu visible à l'histoire réelle un élément nouveau et à contre-courant ${ }^{30} \gg$. Nous habitons au quotidien dans une « soi-disant histoire ${ }^{31} »($ sogenannten Geschichte) que nous découpons temporellement, en laquelle nous reconnaissons des événements, que nous dressons en faits. Ce champ temporel est aussi celui de l'«écart moral ${ }^{32} »$ (moral gap) dans lequel nous faisons l'expérience de la distance entre les obligations que nous nous reconnaissons et notre incapacité naturelle à les assumer. C'est en revanche «l'histoire réelle » (eigentlichen Geschichte), l'histoire que Dieu choisit de partager avec sa créature, qui comble ce fossé entre l'être et le devoir être. Par l'incarnation, l'histoire de Dieu fait irruption dans l'histoire des êtres humains, le Royaume de Dieu s'approche de manière décisive. Au moins à certains endroits du texte, Barth comprend le Royaume de Dieu comme un processus de croissance organique ${ }^{33}$, qui prend racine au cœur d'un monde pourtant encore pécheur, et s'y manifeste progressivement jusqu'à rendre pleinement visible, en fin de compte, la victoire de Dieu.

Ce positionnement théologique n'est pas sans soulever des difficultés. En effet, en laissant espérer une réconciliation dans l'histoire entre le monde et Dieu, il n'est pas certain que Barth se prémunisse entièrement contre le risque d'univocité que nous évoquions plus haut. La croissance du Royaume est certes cachée initialement, mais à mesure qu'elle se développe, elle se révèle toujours davantage également. Dieu se manifeste initialement comme un Dieu caché, mais cette part cachée est amenée à se réduire. On peut donc penser un point de l'histoire où «le voile est levé », c'est-à-dire où le dévoilement de Dieu aura entièrement succédé à son

\footnotetext{
${ }^{29}$ K. BARTH, Der Römerbrief (Erste Fassung) 1919, Zürich, TVZ, 1985, p. 457.

${ }^{30}$ Ibid., p. 85 .

${ }^{31}$ Ibid., p. 67.

32 J. HARE, The Moral Gap: Kantian Ethics, Human Limits, and God's Assistance, Oxford, Clarendon Press, 2002.

${ }^{33}$ B. MCCORMACK, Karl Barth, op. cit., p. 141-155.
} 
voilement (là où, au contraire, la theologia crucis de Luther cherche à penser ensemble le voilement et le dévoilement de Dieu : Dieu se manifeste dans le voilement). Dès lors, la connaissance de Dieu est accessible directement, et il n'y a plus besoin de la ligne brisée de la dialectique pour y parvenir. Dans son effort pour penser Dieu comme au-delà de la contradiction de la thèse et de l'antithèse, Barth en vient à nier l'importance de cette contradiction, à faire comme si tout était déjà joué. Le retour du champ lexical de la lutte, du combat dans la seconde édition du Römerbrief sera alors un moyen de prendre davantage au sérieux la négativité du monde et son altérité radicale par rapport à Dieu. Reconnaître la victoire de Dieu ne doit pas conduire à congédier l'importance de la guerre qui y prépare.

\section{LA LIGNE DE MORT DU RÖMERBRIEF II}

La guerre est présente à l'acmé du Römerbrief de 1922, lorsque Barth commente Rm $12-13$, où il traite du «problème de la morale ${ }^{34} »$. Cette présence au cœur d'un chapitre consacré à la praxis chrétienne concrète pourrait laisser croire, en première lecture, que Barth ne parlerait que de la guerre au sens politique, comme un événement historique conventionnel dans lequel deux États ou plus s'affrontent. Il est vrai que c'est dans ces pages qu'il écarte explicitement et «d'une main ferme » le militarisme, tout comme le pacifisme, «d'une main amène ${ }^{35}$ ». Mais il ne s'agit là que d'une remarque incidente dans une discussion où la guerre prend un sens existential ou ontologique. «La guerre, nous dit Barth, est l'acte naturel de l'homme qui, érigeant en absolu la façon dont $i l$ voit son semblable, veut être comme Dieu ${ }^{36}{ }$. La guerre est une attitude subjective (par exemple, un sentiment d'injustice à l'égard d'une agression qui pousse à prendre les armes) qui se prétend objective, absolue. Un pays se défendra en effet en arguant de critères qui justifient objectivement l'usage de la force. Ce sont ces critères qu'évaluent les théories de la guerre juste, et ils sont en effet censés jouir d'une valeur morale indépendante de l'identité des belligérants. Mais faire ainsi, c'est se mettre à la place de Dieu,

\footnotetext{
${ }^{34}$ K. BARTH, L'Épître aux Romains, op. cit., p. 404.

${ }^{35}$ Ibid., p. 445.

${ }^{36}$ Ibid., p. 444.
} 
« se saisir du sceptre de Dieu ${ }^{37}$ » en faisant justice soi-même pour son propre droit, ce que Barth appelle « la suprême tentation du titanisme ${ }^{38} »$.

Or, l'ennemi lui-même ne procède pas autrement, en en appelant à une justification objective de son propre recours à la force : «C'est là, bel et bien, le mystère de l'« ennemi », que son acte, lui aussi, revient, par nature, à se saisir du spectre de Dieu. L'ennemi, lui aussi, a regretté, en quelque façon, qu'une justice supérieure ne se manifeste pas ${ }^{39}{ }$. Barth ne reprend pas l'argument sceptique classique qui consisterait à dire que tout jugement moral est subjectif, « vérité au-deçà des Pyrénées, erreur au-delà ». Il est prêt à affirmer au contraire qu'en un sens, les belligérants peuvent tous deux prétendre pratiquer objectivement la justice (on peut songer ici à la conviction profonde, commune à la France et à l'Allemagne, de mener une guerre juste, une guerre défensive). Mais le problème est que, ce faisant, ils ne peuvent s'empêcher de prendre sur eux-mêmes une tâche qui est de Dieu. Ils cèdent à « la dernière illusion, selon laquelle pour nous, les hommes, la justice de Dieu serait «praticable » autrement que dans le mal $^{40} \gg$. Ils veulent être des dieux, ils ne sont que des titans. Ils restent prisonniers de la sphère de l'objectivité, qui présente une légitimité en son ordre, mais qui ne respecte pas les distances entre Dieu et l'homme en s'arrogeant la prérogative de l'éternel. L'éthique est nécessairement prométhéenne.

La thèse qui sous-tend ces pages et d'autres semblables est originale ${ }^{41}$ : elle consiste à affirmer que nos évaluations éthiques sont confinées aux limites immanentes de notre expérience. La sphère de l'objectivité morale (l'ensemble de nos justifications qui cherchent à valoir nécessairement et universellement) est valable et nous oblige, certes, mais dans son ordre seulement. Elle n'ouvre pas à une transcendance, elle ne donne aucun accès à la volonté même de Dieu : « Dieu reste libre ${ }^{42} »$. La ligne qui conduirait de nos évaluations morales à ce qui est

\footnotetext{
${ }^{37}$ Ibid., p. 447.

${ }^{38}$ Ibid., p. 446.

${ }^{39}$ Ibid., p. 447.

${ }^{40}$ Ibid.

${ }^{41}$ On la trouverait exprimée sous d'autres formes chez H. BERGSON, Les Deux sources de la morale et de la religion, F. Keck, G. Waterlot (éd.), Paris, PUF, 2008, ainsi que chez E. LevinAS, Totalité et infini: essai sur l'extériorité, La Haye-Boston, Martinus Nijhoff, 1961. Une comparaison plus précise entre les trois auteurs sur ce point reste à faire.
}

${ }^{42}$ K. BARTH, L'Épître aux Romains, op. cit., p. 445 
réellement moral sous le regard de Dieu est une ligne brisée. Toutefois, et c'est là le point remarquable, de cela nous ne nous apercevons pas. Nos évaluations peuvent être objectives, justifiées. Il n'y a pas lieu de sombrer dans le scepticisme ou le relativisme moral. De même, la justice des hommes n'est pas une injustice, elle obéit à une logique de la rétribution qui est une logique, et qui à ce titre est bel et bien porteuse d'une rationalité propre. Mais c'est la justice des hommes et non la justice de Dieu, la rationalité humaine et non le Logos divin. Si Dieu est réellement transcendant au monde — point sur lequel insiste tout le commentaire —, alors il faut le supposer au-delà des contraintes de notre raison et de notre logique.

L'éthique est selon Barth « réfractée » car elle n'émane pas directement de Dieu mais se déploie selon une logique humaine, intramondaine. Afin de préserver le soli Deo gloria, Barth veille à situer Dieu au-delà de toute logique humaine, fut-elle une dialectique complémentaire où la thèse et l'antithèse ne sont pas simplement opposées de manière statique mais se répondent dynamiquement. Pour cela, il propose une dialectique supplémentaire qui admet un étage supérieur, un dépassement (Aufhebung) de la contradiction. Toutefois, contrairement à ses conceptions précédentes, Barth n'admet plus que cette synthèse puisse avoir lieu à la manière d'un processus historique, ou dans l'expérience humaine, mais il la place en Dieu seul. Pour préserver Dieu de toute confusion avec le monde, Barth établit une dialectique à deux niveaux, la contradiction entre le oui et le non d'une part, et son dépassement dans le oui qui n'appartient qu'à Dieu d'autre part. Dieu seul peut franchir la séparation entre le niveau supérieur et le niveau inférieur par la révélation. On peut même considérer que l'opposition de la thèse et de l'antithèse est déjà prise dans le oui de Dieu. Mais cela n'est donné qu'à Dieu. Dans la sphère de la pensée humaine, la dialectique demeure brisée et ne peut prétendre atteindre le niveau supérieur sans se placer elle-même sur le trône de Dieu : «Interrompez-vous dans votre dialectique, afin qu'elle reste dialectique ${ }^{43} »$.

La sphère éthique, la sphère des affaires humaines considérée dans son immanence, est donc placée irrémédiablement sous le signe du Deus absconditus. On comprend alors mieux en quoi l'état de guerre, la conflictualité intramondaine est une expérience indépassable de la condition humaine. Barth renoue en 1922 avec les accents de la theologia crucis, peut-être davantage qu'à aucun autre moment de son œuvre théologique. «Entre Dieu et nous se dresse

\footnotetext{
${ }^{43}$ Ibid., p. 406.
} 
et se dressera jusqu'à la fin des jours la Croix qui unifie, mais aussi qui crée une distance ${ }^{44}$ ». Cette distance se dresse dans l'ordre de l'être $e t$ de la pensée, et pour l'exprimer Barth a recours à la notion de la ligne de mort (Todeslinie) : «Ce que Dieu est et ce que Dieu fait sont et restent une chose, l'être et l'acte de l'homme sont et restent une autre chose. Infranchissable est tracée entre ici et là-bas la ligne de mort ${ }^{45} \gg$. La ligne de mort apparaît à l'être humain comme une faille infranchissable, «la frontière absolue de toutes choses humainement visibles ${ }^{46}$ », un « glaive acéré [qui] pourfend ${ }^{47} »$.

Certes, la ligne de mort n'apparaît comme coupure mortelle que du point de vue de la créature, tandis que vue en Dieu elle est une « ligne de mort, qui, à la vérité, est la ligne de vie, la fin qui est le commencement, le Non qui est le Oui ${ }^{48} \gg$. Mais que la ligne de mort soit en réalité une ligne de vie, c'est précisément cela que le croyant ne peut pas voir. Ce n'est que par les yeux de la foi que la vie du ressuscité est manifestée de manière voilée dans la croix. Ce n'est qu'en poussant jusqu'à son terme logique l'expérience de la mort et de la finitude humaine que l'on aménage l'espace disponible à l'accueil du Christ vivant. La résurrection ne rend donc pas la croix superflue, elle n'en fait ni un contretemps ni un délai : la résurrection intègre indissolublement la croix.

À ce propos, il est frappant de constater que le vocabulaire de la lutte et du combat n'est jamais autant présent que lorsque Barth commente avec Paul le passage, en Christ, du monde ancien à un monde nouveau $(\mathrm{Rm} 5)$. L'homme renouvelé en Jésus Christ n'est pas délivré pour autant du monde ancien :

Aucune unification de Dieu et de l'homme, aucune abolition de la ligne de mort n'a lieu, aucune usurpation anticipée de la plénitude de Dieu, de la délivrance et de la rédemption finale. La rivalité entre l'esprit et la chair, entre la chair et l'esprit, subsiste dans toute son acuité. L'homme reste l'homme, et Dieu reste Dieu ${ }^{49}$.

\footnotetext{
${ }^{44}$ Ibid., p. 109.

${ }^{45}$ Ibid., p. 108.

${ }^{46}$ Ibid., p. 134.

${ }^{47}$ Ibid., p. 231.

${ }^{48}$ Ibid., p. 108.

${ }^{49}$ Ibid., p. 147.
} 
Le chrétien reste soumis aux «tribulations » $(\mathrm{Rm} 5,3)$ en ce monde car la paix qu'il reçoit de Dieu est précisément la paix de Dieu et pas de ce monde ancien. Barth ne mâche pas ses mots pour décrire le degré de conflictualité qui caractérise ce dernier :

Les «tribulations », la détresse de l'homme dans le monde, la «ruine de l'homme extérieur » (II Cor. 4:16), qui s'étend à son essence et à son être les plus intimes, l' « énergie de la mort », que l'apôtre expérimente en lui-même (II Cor. 4:12), les « combats au-dehors, craintes au-dedans », dans lesquels il se trouve (II Cor. 7:5) ${ }^{50}$.

Par conséquent, il ne peut qu'admettre la pleine réalité du mal, qui n'est pas susceptible d'être amoindrie par une théodicée. Plus précisément, cela implique d'admettre la pleine réalité du conflit dialectique lui-même, qui du point de vue humain, ne sera jamais dépassé dans une synthèse. La foi qui veut croire en la promesse de Dieu est réduite à être

une foi au milieu des tribulations, alors qu'on est affligé, et non à côté d'elles, et qui ne survient pas seulement après que ces tribulations, extérieures ou intérieures, ont été heureusement surmontées (überwundenen), adoucies ou, pour finir, endurées (...) Dans la paix de Dieu il existe une souffrance, on s'enfonce, on est perdu et déchiré51.

Le mal demeure une réalité indépassable parce que notre logique est une logique immanente à notre finitude et incapable de se dépasser elle-même dialectiquement. Notre dialectique est brisée, l'accès à l'étage supérieur de la synthèse nous est impossible. Lorsque nous tâchons de le faire, nous ne parvenons pas à sortir de l'immanence de la pensée conceptuelle. Or « la dialectique du salut n'est pas la dialectique hégélienne ${ }^{52} »:$ Dieu n'est pas selon Barth un concept ou une idée, qui obérait donc à une logique quelconque (fut-elle dialectique). Dieu est une réalité en un sens extra-logique : il se manifeste concrètement dans une événement - l'incarnation — qui n'est pas du même ordre qu'une proposition logique. C'est en ce sens que la synthèse en Dieu n'est pas à proprement parler le résultat de la contradiction des deux versants positifs et négatifs de la dialectique. Le oui de Dieu est d'un autre ordre de réalité que le oui et le non de notre dialectique, la synthèse brise le cercle de la thèse et de l'antithèse davantage qu'elle n'en provient.

\footnotetext{
${ }^{50}$ Ibid., p. 150.

${ }^{51}$ Ibid., p. 151.

${ }^{52}$ H. BouILlard, Karl Barth. Genèse et évolution de la théologie dialectique, Paris, Aubier Montaigne, 1957, vol.1, p. 30.
} 
Il y a un corollaire à cette rupture logique et ontologique entre les deux niveaux de la dialectique. C'est que s'il n'y a de synthèse qu'en Dieu, inversement le monde ne peut que demeurer prisonnier de l'expérience de la contradiction entre la thèse et l'antithèse. Cette brisure se jouant non seulement au niveau de la pensée, mais de l'être, on ne peut pas même mettre entre parenthèses la finitude, la congédier ou du moins ne la compter pour rien par rapport à l'éternité du Oui divin. En effet, si cette autonomie ontologique de notre monde visà-vis de Dieu doit être prise au sérieux, alors on ne doit pas s'attendre à ce que les contradictions qui déchirent le monde soient purement et simplement réparées dans l'autre. Il existe une souffrance jusque dans la paix de Dieu, nous a rappelé Barth. Par conséquent, la contradiction est bien pour nous une opposition concrète, réelle, touchant l'être même du croyant, et qui n'est pas jouée d'avance.

C'est précisément sur le caractère concret, intramondain de cette conflictualité que Barth s'appuie pour justifier l'engagement existentiel du chrétien « au cœur de la mêlée, au cœur de la confusion du monde non racheté, laquelle touche l'homme jusqu'en son tréfonds ». Le chrétien ne saurait demeurer dans l'ignorance et l'inaction à l'égard du monde, au nom d'un éventuel dépassement de la contradiction, d'une éventuelle synthèse repoussée aux calendes grecques. À ce propos, on note que le verbe «surmonter» dans le texte ci-dessus traduit l'allemand überwinden et non aufheben. Il ne s'agit donc justement pas d'un dépassement de nature dialectique, en lequel le croyant pourrait placer son espérance. Celui-ci ne peut faire autrement que d'accepter la lutte pied à pied avec le monde : toute tentative de s'échapper à cette conflictualité ou de prétendre qu'elle ne nous touche pas ne serait qu'une forme, non de dépassement dialectique réel, mais de sublimation, qui est l'illusion psychologique du dépassement. À hauteur d'homme, les contradictions ne se dépassent pas dans l'ordre de la pensée, mais dans l'ordre du réel et dans la concrétude de l'engagement.

\section{CONCLUSION}

Nous étions partis de la question du rôle de la guerre dans la pensée du jeune Karl Barth. Pour y répondre, nous avons dû distinguer entre l'expérience historique de la Première Guerre mondiale et la guerre comme une expérience ontologique. Sous le premier aspect, la Grande Guerre joue avant tout un rôle biographique en précipitant la rupture avec la théologie de son temps. Sous le second aspect en revanche, la guerre comme expérience va prendre un rôle théologique plus précis, un rôle existential et même dialectique. 
Tandis que l'expérience de la guerre ne joue initialement qu'une fonction de repoussoir face à l'expérience de Dieu, celle-ci en vient à caractériser la condition humaine hors de la paix de Dieu. Nous n'avons accès en ce monde qu'à une paix « réfractée », qui laisse l'être humain prisonnier de sa finitude et de l'immanence de son éthique. Certes, la foi nous ouvre à la paix de Dieu, mais c'est encore là une paix invisible, dont l'effectivité ne se manifeste qu'en Dieu. Si l'on a pu parler d'une dialectique supplémentaire et téléologique pour définir la théologie barthienne, il ne nous semble pas excessif de parler d'une dialectique « agonistique » dans le Römerbrief II. En effet, la synthèse n'étant possible qu'en Dieu, l'étage inférieur de la dialectique (celui de la contradiction entre la thèse et l'antithèse) est le seul auquel l'être humain ait accès, du moins par ses propres forces. Dans notre relation à Dieu, nous sommes bloqués au rez-de-chaussée si lui-même ne descend pas nous chercher.

Affirmer qu'il n'y a de synthèse, de paix que de Dieu et en Dieu est, d'une part, une façon d'affirmer que Dieu est au-delà de la logique humaine, fut-elle une logique dialectique comme celle de Hegel. Car la «synthèse » que réalise Dieu n'est pas déductible historiquement du heurt de la thèse et de l'antithèse ; au contraire elle rompt avec cette logique : «Ce Oui-là et ce Non-là ne sont pas liés au Oui et au Non des contenus accidentels de notre vie ${ }^{53} »$. Le oui de Dieu inaugure un monde nouveau, qui pour cette raison dépasse le monde ancien, en restant de surcroît préservé de toute confusion avec celui-ci. Mais, d'autre part, sur le plan de l'action humaine, cela entraîne une autre conséquence importante. Aucun acte, aucun discours humain (même religieux) ne peut se prétendre accéder à la synthèse. La lutte ici-bas n'est pas une étape que nous puissions dépasser, en nous contentant de pointer le doigt vers les cieux. Nulle parole humaine n'est au-dessus de la mêlée. L'action humaine n'en devient pas vaine pour autant : elle en devient plus ambigüe, plus faillible. Et c'est cette fragilité qui confère à la sphère des affaires humaines tout son épaisseur morale, son poids ontologique. Puisque Dieu ne descend pas des cieux tous les jours, c'est à nous qu'il revient d'agir, sans aucun privilège ou savoir dont nous disposerions en tant que chrétiens.

L'éloignement de ce Deus absconditus, la radicalité de cette theologia crucis sont d'autant plus frappants qu'ils constituent presque un hapax dans l'œuvre de Barth. C'est dans L'Épître aux Romains de 1922 que Barth associe le plus étroitement le spectacle accablant de la mêlée à l'être même de l'homme. La première édition du Römerbrief est bien plus optimiste quant à la manifestation progressive du Royaume de Dieu dans ce monde. De même, suite à la

\footnotetext{
${ }^{53}$ K. BARTH, L'Épître aux Romains, op. cit., p. 150.
} 
seconde édition, Barth ne cessera d'atténuer le versant critique de sa théologie. Tandis que dans les années 1920 l'être humain se trouve davantage dans le non que dans le oui ${ }^{54}$, c'est l'inverse qui est vrai à partir du début des années 1930. On peut le regretter, au vu de la façon quelque peu mécanique dont la théorie barthienne de la guerre est calquée sur la structure christologique de la Kirchliche Dogmatik ${ }^{55}$. Il est possible qu'une théologie davantage ancrée dans le Römerbrief de 1922 aurait permis de mieux prendre en compte la réalité morale de la guerre. Il nous reste bien des leçons à tirer de ce texte pour notre ontologie et notre compréhension dialectique de l'action de Dieu.

\footnotetext{
${ }^{54}$ K. BARTH, L'Épître aux Romains, op. cit., p. 437.

${ }^{55}$ N. BIGGAR, «Karl Barth's Trinitarian Ethics », in John WeBSTER (dir.), The Cambridge Companion to Karl Barth, Cambridge, Cambridge University Press, pp. 212-227, p. 215-216.
} 